\title{
To brace or not? The answer is "it depends". Preliminary results from BrAIST
}

\author{
Lori Dolan", Stuart Weinstein, the BrAIST Study Group \\ From 11th International Conference on Conservative Management of Spinal Deformities - SOSORT 2014 \\ Annual Meeting \\ Wiesbaden, Germany. 8-10 May 2014
}

\section{Background}

Recent research suggests current indications for bracing in AIS result in significant over-treatment. Many patients are actually at low risk for significant progression. Others present at higher risk will benefit little from bracing.

\section{Aim}

This study developed a simple, yet accurate, model of the risk of significant curve progression in AIS and the risk reduction associated with bracing.

\section{Methods}

Data from 238 BrAIST subjects were used (91 observed, 147 braced). All met current indications for bracing (Cobb $20-40^{\circ}$, Risser $<3$ ) and were followed until reaching a Cobb angle of $>50^{\circ}$

\section{Results}

The overall failure rate was $31 \%$ after bracing and $52 \%$ after observation. Age, gender, Risser, Sanders' digital maturity stage (DMS), curve type and Cobb angle were all associated with outcome. DMS stages were more predictive of failure than Risser grade, even with age in the model. The best-fitting model included the DMS (1-2, 3, or $4+$ ), Cobb angle, and treatment ( $<<0.0001$, c statistic $=0.841$ ). Increasing Cobb angle was associated with increased risk of failure across all DMS's; bracing significantly decreased the risk. In DMS 1-2, the risk of failure ranged from $73 \%\left(\right.$ Cobb $\left.20^{\circ}\right)$ to $93 \%\left(\right.$ Cobb $\left.39^{\circ}\right)$. Bracing reduced the risk to $50 \%$ and $84 \%$, respectively. Risk was lower in DMS 3 patients, ranging from $36 \%\left(\right.$ Cobb $\left.20^{\circ}\right)$ to $76 \%\left(\right.$ Cobb $\left.40^{\circ}\right)$, reducing to $19 \%$ to $52 \%$ with bracing. The lowest risk of failure was noted at DMS 4+, ranging

University of lowa, lowa City, IA, USA 